ARTICLE

\title{
Spin-orbit quantum impurity in a topological magnet
}

\author{
Jia-Xin Yin (1) 1,11凶 , Nana Shumiya ${ }^{1,11}$, Yuxiao Jiang 1,11, Huibin Zhou 2,11, Gennevieve Macam (1) 3,11, \\ Hano Omar Mohammad Sura (1) 4,11, Songtian S. Zhang (1) 1, Zi-Jia Cheng1, Zurab Guguchia (1), Y, Yangmu Li® ${ }^{6}$, \\ Qi Wang7, Maksim Litskevich (1) 1, Ilya Belopolski', Xian P. Yang (1) 1, Tyler A. Cochran', Guoqing Chang (1) 1, \\ Qi Zhang ${ }^{1}$, Zhi-Quan Huang ${ }^{3}$, Feng-Chuan Chuang (10 ${ }^{3}$, Hsin Lin (10) ${ }^{8}$, Hechang Lei (1) ${ }^{7}$, Brian M. Andersen ${ }^{4}$, \\ Ziqiang Wang9, Shuang Jia (1) ${ }^{2} \&$ M. Zahid Hasan (10) 1,10凶
}

Quantum states induced by single-atomic impurities are at the frontier of physics and material science. While such states have been reported in high-temperature superconductors and dilute magnetic semiconductors, they are unexplored in topological magnets which can feature spin-orbit tunability. Here we use spin-polarized scanning tunneling microscopy/ spectroscopy (STM/S) to study the engineered quantum impurity in a topological magnet $\mathrm{Co}_{3} \mathrm{Sn}_{2} \mathrm{~S}_{2}$. We find that each substituted In impurity introduces a striking localized bound state. Our systematic magnetization-polarized probe reveals that this bound state is spindown polarized, in lock with a negative orbital magnetization. Moreover, the magnetic bound states of neighboring impurities interact to form quantized orbitals, exhibiting an intriguing spin-orbit splitting, analogous to the splitting of the topological fermion line. Our work collectively demonstrates the strong spin-orbit effect of the single-atomic impurity at the quantum level, suggesting that a nonmagnetic impurity can introduce spin-orbit coupled magnetic resonance in topological magnets.

\footnotetext{
${ }^{1}$ Laboratory for Topological Quantum Matter and Advanced Spectroscopy (B7), Department of Physics, Princeton University, Princeton, NJ 08544, USA.

${ }^{2}$ International Center for Quantum Materials and School of Physics, Peking University, 100193 Beijing, China. ${ }^{3}$ Department of Physics, National Sun Yat-sen University, Kaohsiung 80424, Taiwan. ${ }^{4}$ Niels Bohr Institute, University of Copenhagen, Universitetsparken 5, DK-2100 Copenhagen, Denmark. ${ }^{5}$ Laboratory for Muon Spin Spectroscopy, Paul Scherrer Institute, CH-5232 Villigen, PSI, Switzerland. ${ }^{6}$ Condensed Matter Physics and Materials Science Division, Brookhaven National Laboratory, Upton, NY 11973, USA. ${ }^{7}$ Department of Physics and Beijing Key Laboratory of Opto-electronic Functional Materials \& Micro-nano Devices, Renmin University of China, 100872 Beijing, China. ${ }^{8}$ Institute of Physics, Academia Sinica, Taipei 11529, Taiwan. ${ }^{9}$ Department of Physics, Boston College, Chestnut Hill 02467 MA, USA. ${ }^{10}$ Materials Sciences Division, Lawrence Berkeley National Laboratory, Berkeley, CA 94720, USA. ${ }^{11}$ These authors contributed equally: Jia-Xin Yin, Nana Shumiya, Yuxiao Jiang, Huibin Zhou, Gennevieve Macam, Hano Omar Mohammad Sura.

凶email: jiaxiny@princeton.edu; mzhasan@princeton.edu
} 
U nderstanding the single-atomic impurity state in a quantum material is a fundamental problem with widespread implications in physics and technology ${ }^{1-8}$. For instance, the $\mathrm{Zn}$ impurity state in a high-temperature superconductor uncovers the Cooper pairing symmetry ${ }^{3}$, the $\mathrm{Mn}$ impurity state in a semiconductor elucidates the ferromagnetic coupling 4 , and the interstitial $\mathrm{Fe}$ impurity in a superconductor with topological surface states creates Majorana-like state ${ }^{5}$. Besides being a local probe of the quantum materials, the impurity state with discrete or (magnetic) bistable quantum levels is valuable for the quantum technology ${ }^{6-8}$. Most known single-atomic impurity states are, however, either from the spin or orbital channel, limiting their tunability at the quantum level. Recently, spin-orbit coupled kagome magnets have emerged as a new class of quantum materials suitable for microscopic research ${ }^{9-19}$. In particular, we notice that the In doped $\mathrm{Co}_{3} \mathrm{Sn}_{2} \mathrm{~S}_{2}$ exhibits strongly altered bulk magnetic and transport properties, including reductions of magnetism, suppressions of metallicity, and variations of anomalous Hall conductivity ${ }^{20-22}$. These effects imply a striking, yet not understood quantum state associated with each nonmagnetic In impurity in this topological magnet. Therefore, a single crystal of $\mathrm{Co}_{3} \mathrm{Sn}_{2} \mathrm{~S}_{2}$ containing a dilute concentration of In impurities is considered a natural and promising quantum material for experiments on atomic impurity state with spin-orbit tunability. Here we report our spin-polarized STM/S study of $1 \%$ In doped $\mathrm{Co}_{3} \mathrm{Sn}_{2} \mathrm{~S}_{2}$, which uncovers a spin-orbit quantum impurity state.

\section{Results}

Engineered atomic impurity. $\mathrm{Co}_{3} \mathrm{Sn}_{2} \mathrm{~S}_{2}$ has a layered crystal structure and a ferromagnetic ground state (Curie temperature, $\left.T_{\mathrm{C}}=170 \mathrm{~K}\right)$ with the $c$-axis magnetization arising from the Co kagome lattice. Cleaving preferentially breaks its S-Sn bond, which leads to the S and Sn terminated surfaces. Previous STM studies have dominantly observed two surfaces, one with largely vacancy defects and the other with adatom defects ${ }^{11,13,14}$. Several factors challenge their assignation, as the two surfaces have identical lattice symmetries, their interlayer distance is sub- $\AA$ in scale, and STM topographic image convolutes the spatial variation of the integrated local density of states and the geometrical corrugations ${ }^{23}$. Decisive experimental evidence for surface identification can be found by imaging the symmetry-dictated surface boundary and the layer-selective chemical dopants ${ }^{23-25}$. In previous work ${ }^{11}$, we have determined that the vacancy surface is the Sn layer and the adatom surface is the $S$ layer by imaging their surface boundary determined by the crystalline symmetry. We further conclude this assignation by doping the bulk $\mathrm{Co}_{3} \mathrm{Sn}_{2} \mathrm{~S}_{2}$ single crystals with $1 \%$ In impurities and imaging the layerselective In-dopants. The In impurities preferentially replace the Sn atoms according to previous experimental and theoretical studies $^{20,21}$, as well as our recent systematic single-crystal growth $^{22}$. Indeed, on the vacancy surface identified ${ }^{11}$ to be $\mathrm{Sn}$, we observe dilute substitutional atoms with consistent concentration (Fig. 1a), suggesting these impurities to be In atoms.

Spatial feature of single impurity resonance. By performing an extensive study on the electronic properties of the In impurities, we find that each impurity repeatedly features a sharp state at the negative energy as shown in Fig. 1b. First-principles calculations show that each In impurity is almost nonmagnetic but introduces a strong resonance (Fig. 1c), similar to the experimental data. The calculations further reveal that the impurity resonance arises from a spin-down state (opposite to the bulk magnetization direction) and resides in the spin-down bandgap. Thus, the magnetic resonance state likely arises from the local impurity perturbation of the spin-polarized band structure. As the low-energy band structure is dominated by Co $3 d$ orbitals, the resonant impurity state of the In atom also implies that there a strong local impact on the Co kagome lattice in real-space. To explore the detailed
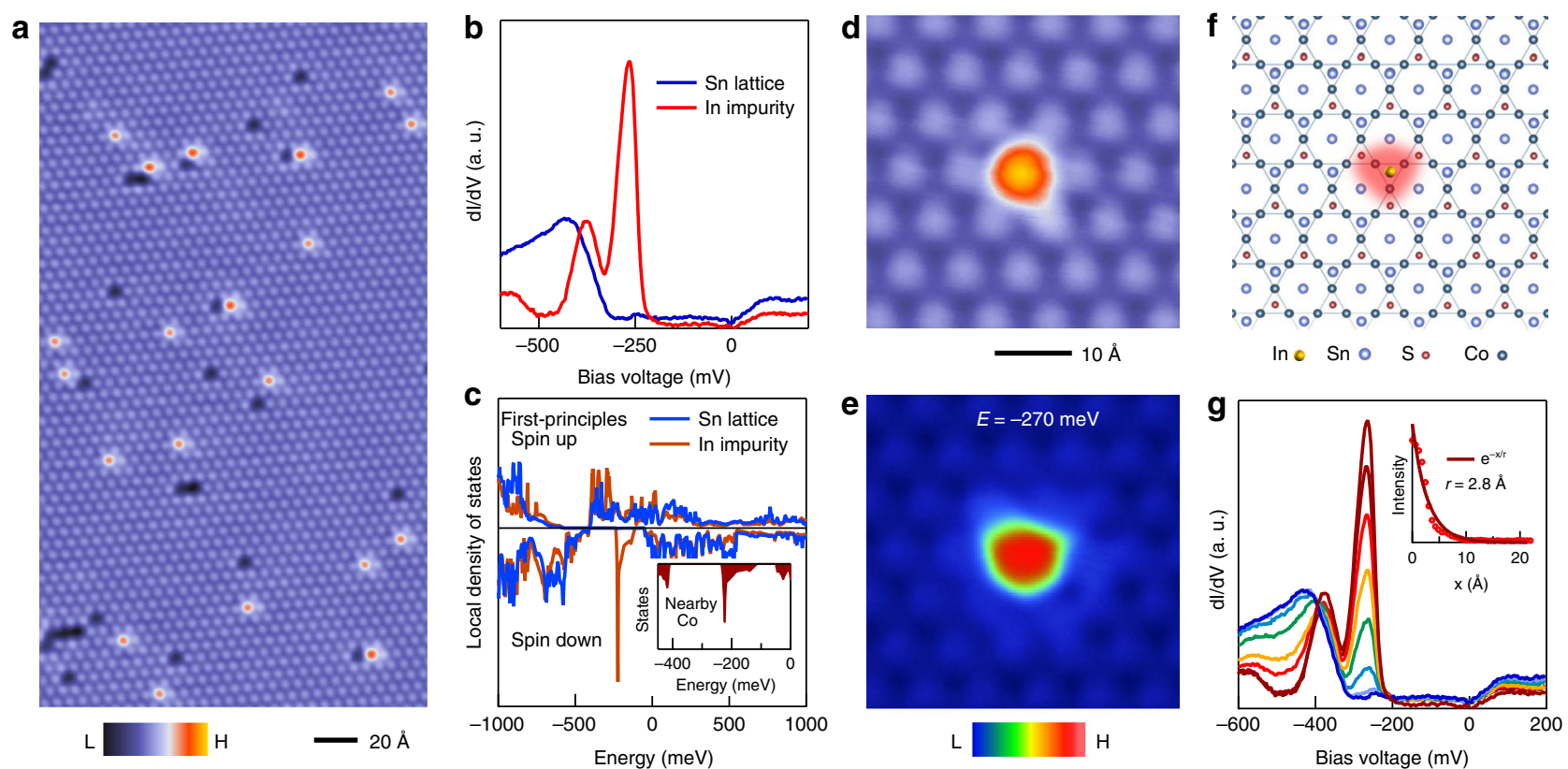

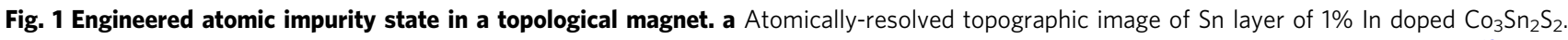
b Differential conductance spectrums taken on the Sn lattice (blue) and at the In impurity (red), respectively. c First-principles calculation ${ }^{13}$ of the spinresolved local density of states of the Sn lattice (blue) and an In impurity (red), which shows a magnetic impurity resonance. The inset shows the spindown states of the Co atom closest to the In impurity. $\mathbf{d}$ Topographic image of an isolated impurity. e Corresponding differential conductance map taken at $E=-270 \mathrm{meV}$ (resonance energy). $\mathbf{f}$ Correlation between the atomic structure and the pattern in the differential conductance map. $\mathbf{g}$ Differential conductance spectra taken across the surface with spatial variation from the center of the In impurity (dark red) to far away (blue). The inset shows the exponential fit to the spatial decay of the impurity resonance. 
real-space feature, we probe the local electronic structure for an isolated In impurity, as shown in Fig. 1d. The corresponding dI/ $\mathrm{dV}$ map at the impurity resonance energy in Fig. 1e shows a localized pattern bound to the impurity site (Fig. 1e). The bound state couples with three nearby Co atoms in the underlying kagome lattice, as illustrated in Fig. 1f. This is consistent with the first-principles calculation that the nearby magnetic Co atoms also feature such resonance state (inset of Fig. 1c), supporting the Co-In coupling (See Supplementary Note 1). Figure 1g shows the representative $\mathrm{d} I / \mathrm{d} V$ curves measured with increasing distance from the impurity, demonstrating the bound state decaying in intensity without detectable energy dispersion or splitting. An exponential fit to the decay yields a characteristic length scale of $2.8 \AA$ (inset of Fig. 1g). Therefore, these systematic characterizations reveal that the nonmagnetic In impurity couples with the underlying magnetic kagome lattice to introduce a striking localized bound state.

Magnetic nature of single impurity resonance. To probe the magnetic nature of the impurity bound state, we perform tunneling experiments with a spin-polarized $\mathrm{Ni}$ tip under weak magnetic fields ${ }^{26-29}$. The bulk crystal has a coercive field $B_{\mathrm{C}} \sim 0.3$ $\mathrm{T}$, and Ni tip is a soft magnet with a $B_{\mathrm{C}} \ll 0.1 \mathrm{~T}$ that can be easily flipped by reversing the magnetic field ${ }^{29}$. We measure the tunneling signal of the impurity state while sequentially applying fields along the $c$-axis of $+0.5 \mathrm{~T},+0.1 \mathrm{~T},-0.1 \mathrm{~T},-0.5 \mathrm{~T},-0.1 \mathrm{~T}$, and $+0.1 \mathrm{~T}$ to systematically flip the magnetization of the tip and sample (Fig. 2a). This sequence allows us to perform spinpolarized measurements of the impurity. The $+0.5 \mathrm{~T}$ field polarizes both the sample and tip, aligning the spin of the tip and anti-aligning the spin of the impurity state, due to the spin-down nature of the impurity state. $\mathrm{A}+0.1 \mathrm{~T}$ field does not change the polarization of either the tip or impurity state. Flipping the field to $-0.1 \mathrm{~T}$ also flips the spin of the tip, leaving the spin of the impurity state unchanged (down). Here, with both tip and impurity state spins aligned down, we observe an intensity increment of the tunneling signal. Next, we further decrease the field to $-0.5 \mathrm{~T}$, which flips up the spin of the impurity state with a corresponding reduction of the tunneling signal. Last, by sequentially changing the field to $-0.1 \mathrm{~T}$ and $+0.1 \mathrm{~T}$, we flip the spin of the tip (down) and again observe an increase in the intensity. Our progressive field manipulation strongly supports that impurity state features spin-down polarization tied to the bistable magnetic bulk, consistent with the first-principles calculation.

To further determine the effective moment of this magnetic polarized state, we probe the state by applying a strong external magnetic field $\left(|\mathbf{B}| \gg B_{\mathrm{C}}\right)$ along the $c$-axis with a nonmagnetic tip. Under the field, a spin-up/spin-down band hosting an intrinsic magnetic moment of $+1 /-1$ Bohr magneton will exhibit a Zeeman shift to lower/higher energies in a rate of $0.058 \mathrm{meVT}^{-1}$. Moreover, when the magnetism of the system is polarized with an applied field, the spin-polarized state will always shift to the same energy direction regardless of the relative field orientation ${ }^{11}$ (top inset schematic in Fig. 2b), which was also experimentally observed for the $8 \mathrm{~T}$ and $-8 \mathrm{~T}$ data (Fig. $2 \mathrm{~b}$ ). The positive energy shift indicates the state has a negative magnetic moment, calculated to be $-5 \mu_{\mathrm{B}}$ (or a Landé $g$ factor of 10) based on a shift rate of $0.275 \mathrm{meVT}^{-1}$ (right inset of Fig. 2b). This large value is beyond the spin Zeeman effects $\left(\sim-1 \mu_{\mathrm{B}}\right)$ and indicates the additional negatively polarized orbital magnetization. The anomalous Zeeman effect with an unusual moment or $g$ factor has been observed in the electronic bands of kagome magnets $9,11,17$, which is often linked to the Berry phase physics associated with magnetism and spin-orbit coupling ${ }^{9,11,30,31}$. Since the In impurity couples with the Co atoms in the kagome lattice, the higher orbital angular momentum of the hybridized In-Co orbital can contribute to the large effective moment. We note that the effective moment of $-5 \mu_{\mathrm{B}}$ represents the diamagnetic response induced by the In impurity, and should not be thought as the local magnetic moment of the impurity atom.

Interacting impurity states induced quantized orbitals. Having characterized magnetic resonance state from the isolated impurity, we further probe the coupled impurity states to understand how they interact with each other through extensive imaging and spectroscopy investigation with a nonmagnetic tip. In Fig. 3a, we present the evolution of the impurity bound state with increasing perturbation strength caused by a second nearby impurity. We find that with decreasing spatial separation, the bound state progressively decreases in intensity and finally splits into two subpeaks. Figure $3 \mathrm{~b}$ further compares three cases with one isolated impurity, two neighboring impurities, and three neighboring impurities, respectively. We find the quantized number of split impurity states matches with the coupled impurity number, highlighting their atomic-scale quantum-level
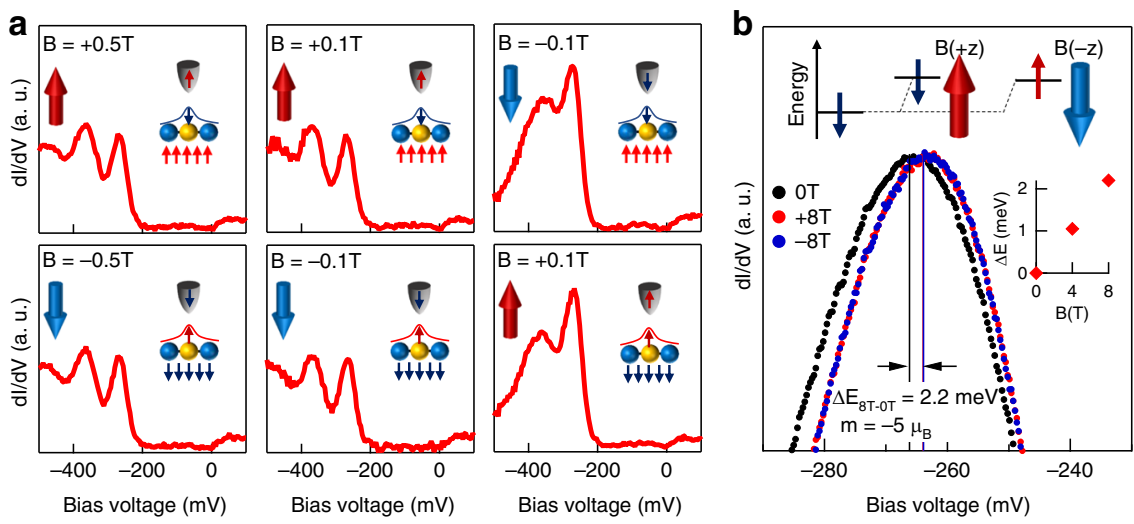

Fig. 2 Magnetic nature of the impurity bound state. a Dependence of the impurity state with Ni tip under a weak magnetic field. We apply $+0.5 \mathrm{~T}$, $+0.1 \mathrm{~T}$, $-0.1 \mathrm{~T},-0.5 \mathrm{~T},-0.1 \mathrm{~T},+0.1 \mathrm{~T}$ fields to systematically flip the magnetization of the tip and sample. The inset schematics illustrate the respective spins of the tip and the impurity state that is anti-aligned with bulk magnetization direction. $\mathbf{b}$ Dependence of the impurity state with a strong magnetic field. Under both $+8 \mathrm{~T}$ and $-8 \mathrm{~T}$, the peak exhibits a magnetization-polarized Zeeman energy shift of $2.2 \mathrm{meV}$, which amounts to an effective moment of $-5 \mu_{\mathrm{B}}$. The inset data shows the energy shift for different magnetic field magnitudes. Inset schematic illustrates the magnetization-polarized Zeeman effect. The applied field aligns the spins of the impurity state in the same orientation, hence $+z$ and $-z$ orientation fields lead the energy to shift in the same direction. 

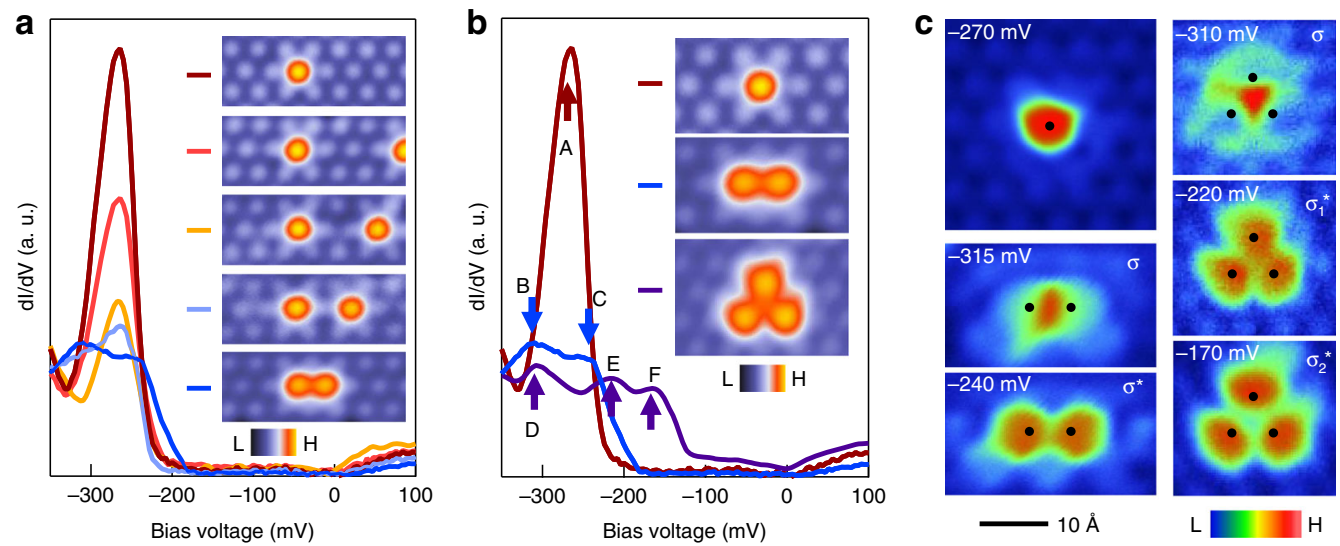

Fig. 3 Interacting impurity states induced quantized orbitals. a Differential conductance spectra taken at the central impurity with perturbations of varying strengths from a second impurity. Inset: respective topographic images for impurity configurations. Note the images are acquired by finding different surface locations. b Local impurity state with coupling to different numbers of impurities. The arrows highlight the quantized splitting with additional interacting impurity numbers. Note the images are acquired by finding different surface locations. c dl/dV maps at the respective bound state energies in (b). $\mathrm{dl} / \mathrm{dV}$ maps taken at $\mathrm{V}=-270 \mathrm{mV}$ for a single impurity; $-315 \mathrm{mV}$ (bonding state $\sigma$ ) and $-240 \mathrm{mV}$ (antibonding state $\sigma$ ) for a double impurity, respectively; $-310 \mathrm{mV}$ (bonding state $\sigma$ ), $-220 \mathrm{mV}$ (antibonding state $\sigma_{1}{ }^{\star}$ ) and $-170 \mathrm{mV}$ (antibonding state $\sigma_{2}{ }^{\star}$ ) for a triple impurity, respectively. The black dots mark the center of impurities.

coupling. Differential conductance maps at these corresponding splitting energies demonstrate their distinct orbital hybridizations (Fig. 3c). For two neighboring impurities, the $\mathrm{d} I / \mathrm{d} V$ maps show a bonding $(\sigma)$ and antibonding $\left(\sigma^{*}\right)$ orbital formation ${ }^{4}$, consistent with the quantum coupling of doubly degenerate states. For three impurities, the $\mathrm{dI} / \mathrm{dV}$ maps show the formation of one bonding $(\sigma)$ and two antibonding $\left(\sigma_{1}{ }^{*}, \sigma_{2}{ }^{*}\right)$ orbitals, an unusual situation in which we discuss below.

\section{Discussion}

Geometrically, the three neighboring impurities have $\mathrm{C}_{3 \mathrm{~V}}$ symmetry, which would form a doubly degenerate orbital state $\sigma^{*}$ protected by the mirror symmetry. The mirror symmetry operation, however, would transfer spin-up to spin-down. On the other hand, the magnetic resonances induced by different nonmagnetic In impurities are expected to be of the same spinpolarization direction, which is locked by the ferromagnetic ordering of the underlying magnetic Co kagome lattice perturbed by these impurities (See Supplementary Note 2). Therefore, the combination of the same spin-polarization direction of the degenerate impurity states and the atomic spin-orbit coupling naturally breaks the mirror symmetry, leading to the splitting of $\sigma^{*}$ (see "Methods" for theoretical modeling). Such splitting is analogous to the splitting of the bulk topological nodal line or magnetic Weyl fermion line $e^{32,33}$, which is protected by the crystalline mirror symmetry.

In conclusion, we report the first STM/S studies of the nonmagnetic impurity behavior in a topological magnet. Associated with the atomic nonmagnetic impurities, we find an intense spinorbit polarized bound state with an unusual magnetic moment and quantized energy splitting under impurity-impurity interaction. The striking spin-orbit quantum states revealed here can advance the understanding of the magnetic and transport behaviors of doped topological magnets, and call for new perspectives on the interplay between magnetism and spin-orbit coupling at the atomic scale. The discrete quantum level of interacting impurities resembles that of a quantum dot, which is critical to nanophotonics and quantum information processing. With the single-atom precision, the atomic quantum dot has a high level of digital fidelity ${ }^{7}$. The interacting spin-orbit polarized quantum impurity involves multiple degrees of freedom, including charge, spin, and orbital, the quantum control of which in the magnetic bistable platform can provide a useful guideline for the development of spin-orbit entangled quantum technology. Note added in proof: with completion of this work, we became aware of Ref. 29, which reported another kind of impurity resonance in $\mathrm{Co}_{3} \mathrm{Sn}_{2} \mathrm{~S}_{2}$ exhibiting negative magnetism as well.

\section{Methods}

STM/S measurement. Single crystals ${ }^{22}$ of $\mathrm{Co}_{3} \mathrm{~S}_{2-\mathrm{x}} \mathrm{In}_{\mathrm{x}} \mathrm{Sn}_{2}$ up to $1.5 \mathrm{~mm} \times 1.5$ $\mathrm{mm} \times 0.3 \mathrm{~mm}$ were used in this study. Samples were cleaved mechanically in situ at $77 \mathrm{~K}$ in ultra-high vacuum conditions, and then immediately inserted into the STM head, already at $\mathrm{He} 4$ base at $4.2 \mathrm{~K}$. The magnetic field was applied under zero-field cooling, after which we carefully approached the tip to locate the same atomic-scale area for tunneling spectroscopy 11,13 . Tunneling conductance spectra were obtained using standard lock-in amplifier techniques with a root mean square oscillation voltage of $0.2 \mathrm{meV}-5 \mathrm{meV}$ and a lock-in frequency of $973 \mathrm{~Hz}$. The topographic images were taken with tunneling junction set up: $V=-300 \sim-500 \mathrm{mV}, I=100$ $\mathrm{pA}$, the conductance maps were taken with tunneling junction set up: $V=-100 \sim$ $-500 \mathrm{mV}, I=200 \mathrm{pA}$, and the tunneling spectra were taken with junction set up: $V=-600 \mathrm{mV}, I=300 \mathrm{pA}$. Commercial STM Ir/Pt tip (nonmagnetic) and STM Ni tip (soft magnet) tips were used in this study. To study the impurity-impurity interaction, we checked the topographic images for over 3000 impurities to obtain the cases in Fig. 3 in the 1\% In doped samples.

Evidence for surface identification. STM studies of $\mathrm{Co}_{3} \mathrm{Sn}_{2} \mathrm{~S}_{2}$ often encounter two kinds of surfaces, one dominated by adatom defects and the other dominated by vacancy defects. Here we discuss our evidence for their assignation ${ }^{11}$ as S surface and Sn surface, respectively. First, as illustrated in Fig. 4a, based on crystalline symmetry, when $S$ and $S n$ surfaces meet at a step edge, the $S n$ surface will be just above the $\mathrm{S}$ surface. This experimental evidence is directly provided in Fig. 4b, where we observe the vacancy surface is above the adatom surface. Second, this identification provides a natural explanation for the origin of the surface defects. The $S n$ vacancy in the $S$ surface and $S n$ adatom on the $S$ surface are simultaneously created due to incomplete cleaving, as seen in the experiment (Fig. 4b). Third, we show that the first-principles calculation of the surface dependent local density of states matches the experimental data (Fig. 4c). Last, we observe In dopant in the vacancy surface in the main paper, and In is known to substitute the Sn atom both experimentally and theoretically ${ }^{20-22}$.

First-Principles calculations. First-principles calculations were performed in the density functional theory ${ }^{34,35}$ framework as implemented in the Vienna Ab initio Simulation Package ${ }^{36,37}$. Generalized gradient approximation in Perdew-Burke -Ernzerhof functional ${ }^{38}$ was applied to describe electron exchange-correlation interaction with the projector augmented wave potentials ${ }^{39}$. The supercell consists of a periodically repeating $2 \times 2$-slab with a thickness of twice the bulk and a vacuum space of $\sim 14 \AA$ along the $z$-direction. The slab is cleaved to reveal the 
a

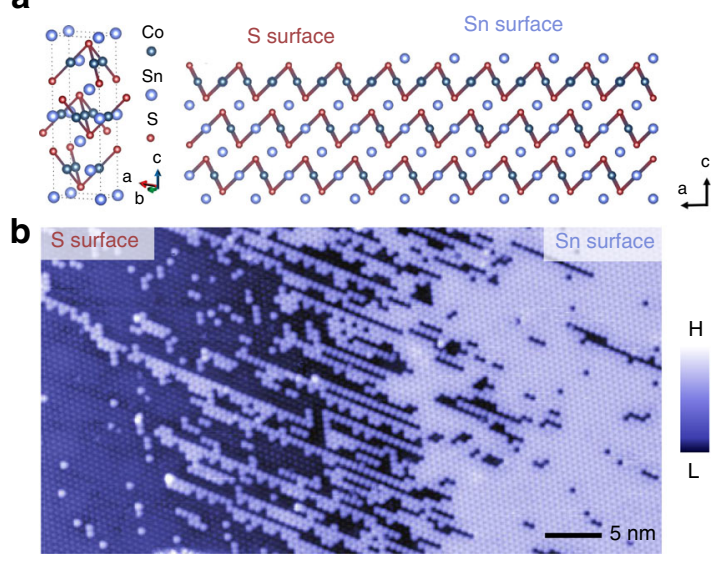

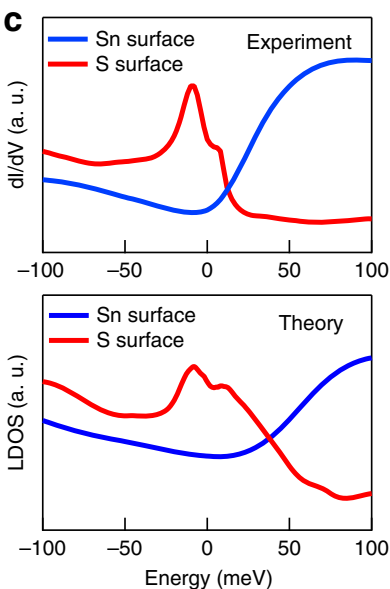

Fig. 4 Extended evidence for surface identification in $\mathbf{C o}_{3} \mathbf{S n}_{\mathbf{2}} \mathbf{S}_{\mathbf{2}}$. a Crystal structure of $\mathrm{Co}_{3} \mathrm{Sn}_{2} \mathrm{~S}_{2}$ (left) and the cleaving surface illustration (right). b Atomically resolved topographic image of the boundary between $S$ surface and Sn surface. The $S$ surface smoothly evolves into the Sn surface with increasing coverage of $\mathrm{Sn}$ adatom. c Comparison between spatially averaged surface dependent dl/dV curves with the first-principles calculations.

Sn-terminating surface, and one surface $\mathrm{Sn}$ atom is replaced with In to simulate dilute doping ( 2.3\%). The energy cutoff was set at $400 \mathrm{eV}$ and the energies in selfconsistent calculations were converged until $10^{-4} \mathrm{eV}$. The Brillouin zone was sampled using a $6 \times 6 \times 1$ Monkhorst-Pack ${ }^{40}$ grid.

\section{Data availability}

All relevant data are available from the corresponding authors upon reasonable request.

Received: 14 May 2020; Accepted: 24 July 2020;

Published online: 04 September 2020

\section{References}

1. Keimer, B. \& Moore, J. E. The physics of quantum materials. Nat. Phys. 13, 1045-1055 (2017).

2. Soumyanarayanan, A., Reyren, N., Fert, A. \& Panagopoulos, C. Emergent phenomena induced by spin-orbit coupling at surfaces and interfaces. Nature 539, 509-517 (2016).

3. Pan, S. H. et al. Imaging the effects of individual zinc impurity atoms on superconductivity in $\mathrm{Bi}_{2} \mathrm{Sr}_{2} \mathrm{Ca}(\mathrm{Cu}, \mathrm{Zn})_{2} \mathrm{O}_{8+\delta}$. Nature 403, 746-750 (2000).

4. Kitchen, D., Richardella, A., Tang, J.-M., Flatte, M. E. \& Yazdani, A. Atom-byatom substitution of $\mathrm{Mn}$ in GaAs and visualization of their hole-mediated interactions. Nature 442, 436-439 (2006).

5. Yin, J.-X. et al. Observation of a robust zero-energy bound state in iron-based superconductor Fe(Te,Se). Nat. Phys. 11, 543-546 (2015).

6. Fuechsle, M. et al. A single-atom transistor. Nat. Nanotechnol. 7, 242-246 (2012).

7. Folsch, S. et al. Quantum dots with single-atom precision. Nat. Nanotechnol. 9, 505-508 (2014).

8. Natterer, F. D. et al. Reading and writing single-atom magnets. Nature 543, 226-228 (2017).

9. Yin, J.-X. et al. Giant and anisotropic spin-orbit tunability in a strongly correlated kagome magnet. Nature 562, 91-95 (2018).

10. Lin, Z. et al. Flatbands and emergent ferromagnetic ordering in $\mathrm{Fe}_{3} \mathrm{Sn}_{2}$ kagome lattices. Phys. Rev. Lett. 121, 096401 (2018).

11. Yin, J.-X. et al. Negative flat band magnetism in a spin-orbit-coupled correlated kagome magnet. Nat. Phys. 15, 443-448 (2019).

12. Li, Y. et al. Magnetic-field control of topological electronic response near room temperature in correlated kagome magnets. Phys. Rev. Lett. 123, 196604 (2019).

13. Jiao, L. et al. Signatures for half-metallicity and nontrivial surface states in the kagome lattice Weyl semimetal $\mathrm{Co}_{3} \mathrm{Sn}_{2} \mathrm{~S}_{2}$. Phys. Rev. B 99, 245158 (2019).

14. Morali, N. et al. Fermi-arc diversity on surface terminations of the magnetic Weyl semimetal $\mathrm{Co}_{3} \mathrm{Sn}_{2} \mathrm{~S}_{2}$. Science 365, 1286-1291 (2019).

15. Yang, H. et al. Scanning tunneling microscopy on cleaved $\mathrm{Mn}_{3} \mathrm{Sn}(0001)$ surface. Sci. Rep. 9, 9677 (2019).

16. Guguchia, Z. et al. Tunable anomalous Hall conductivity through volume-wise magnetic competition in a topological kagome magnet. Nat. Commun. 11, 559 (2020).
17. Yin, J.-X. et al. Quantum-limit Chern topological magnetism in $\mathrm{TbMn}_{6} \mathrm{Sn}_{6}$. Nature 583, 533-536 (2020)

18. Zhang, S. S. et al. Many-body Resonance in a correlated topological kagome antiferromagnet. Phys. Rev. Lett. 125, 046401 (2020).

19. Yin, J.-X. et al. Fermion-boson many-body interplay in a frustrated kagome paramagnet. Nat. Commun. 11, 4003 (2020).

20. Weihrich, R. \& Anusca, I. Half antiperovskites. III crystallographic and electronic structure effects in $\mathrm{Sn}_{2}-\mathrm{xInxCo}_{3} \mathrm{~S}_{2}$. Z. Anorg. Allg. Chem. 632, 1531 (2006).

21. Corps, J. et al. Interplay of metal-atom ordering, Fermi level tuning, and thermoelectric properties in cobalt shandites $\mathrm{Co}_{3} \mathrm{M}_{2} \mathrm{~S}_{2}(\mathrm{M}=\mathrm{Sn}, \mathrm{In})$. Chem. Mater. 27, 3946-3956 (2015).

22. Zhou, H. et al. Enhanced anomalous Hall effect in the magnetic topological semimetal $\mathrm{Co}_{3} \mathrm{Sn}_{2}$-xInxS 2 . Phys. Rev. B 101, 125121 (2020).

23. $\mathrm{Li}, \mathrm{A}$. et al. Surface terminations and layer-resolved tunneling spectroscopy of the 122 iron pnictide superconductors. Phys. Rev. B 99, 134520 (2019).

24. Guan, S.-Y. et al. Superconducting topological surface states in the noncentrosymmetric bulk superconductor $\mathrm{PbTaSe}_{2}$. Sci. Adv. 2, e1600894-e1600894 (2016)

25. Aynajian, P. et al. Visualizing heavy fermions emerging in a quantum critical Kondo lattice. Nature 486, 201-206 (2012).

26. Wiesendanger, R. Spin mapping at the nanoscale and atomic scale. Rev. Mod. Phys. 81, 1495 (2009).

27. Loth, S., Lutz, C. P. \& Heinrich, A. J. Spin-polarized spin excitation spectroscopy. N. J. Phys. 12, 125021 (2010).

28. Focko Meier et al. Revealing magnetic interactions from single-Atom magnetization curves. Science 320, 82-86 (2008).

29. Yuqing Xing, et al. Localized spin-orbit polaron in magnetic Weyl semimetal $\mathrm{Co}_{3} \mathrm{Sn}_{2} \mathrm{~S}_{2}$. Preprint at https://arxiv.org/abs/2001.11295 (2020).

30. Yazyev, O. V. An upside-down magnet. Nat. Phys. 15, 424-425 (2019).

31. Vanderbilt, David. Berry Phases in Electronic Structure Theory: Electric Polarization, Orbital Magnetization and Topological Insulators. (Cambridge University Press, 2018).

32. Hasan, M. Z., Xu, S.-Y. \& Bian, G. Topological insulators, topological superconductors and Weyl fermion semimetals: discoveries, perspectives and outlooks. Phys. Scripta 164, 014001 (2015).

33. Belopolski, I. et al. Discovery of topological Weyl fermion lines and drumhead surface states in a room temperature magnet. Science 365, 1278-1281 (2019).

34. Hohenberg, P. \& Kohn, W. Inhomogeneous electron gas. Phys. Rev. 136, B864 (1964).

35. Kohn, W. \& Sham, L. J. Self-consistent equations including exchange and correlation effects. Phys. Rev. 140, A1133 (1965).

36. Kresse, G. \& Hafner, J. Ab initio molecular dynamics for liquid metals. Phys. Rev. B 47, 558 (1993).

37. Kresse, G. \& Furthmüller, J. Efficient iterative schemes for ab initio totalenergy calculations using plane-wave basis set. Phys. Rev. B 54, 11169 (1996).

38. Perdew, J. P., Burke, K. \& Ernzerhof, M. Generalized gradient approximation made simple. Phys. Rev. Lett. 77, 3865 (1996).

39. Kresse, G. \& Joubert, D. From ultrasoft pseudopotentials to the projector augmented-wave method. Phys. Rev. B 59, 1758 (1999).

40. Monkhorst, H. J. \& Pack, J. D. Special points for Brillouin-zone integrations. Phys. Rev. B 13, 5188-5192 (1976). 


\section{Acknowledgements}

We thank Z. Song, T. Neupert, B. Lian, and H. J. Gao for insightful discussions. Experimental and theoretical work at Princeton University was supported by the Gordon and Betty Moore Foundation (Grant No. GBMF4547 and GBMF9461/Hasan). Sample characterization was supported by the United States Department of Energy (US DOE) under the Basic Energy Sciences programme (Grant No. DOE/BES DE-FG-02-05ER46200). M.Z.H. acknowledges support from Lawrence Berkeley National Laboratory and the Miller Institute of Basic Research in Science at the University of California, Berkeley in the form of a Visiting Miller Professorship. This work benefited from partial lab infra-structure support under NSF-DMR-1507585. M. Z. H. also acknowledges visiting scientist support from IQIMat the California Institute of Technology. The work at Peking University was supported by the National Natural Science Foundation of China No. U1832214, No.11774007, the National Key R\&D Program of China (2018YFA0305601), and the strategic Priority Research Program of Chinese Academy of Sciences (XDB28000000). The work at Renmin University was supported by the National Key R\&D Program of China (Grants No. 2016YFA0300504 and 2018YFE0202600), the National Natural Science Foundation of China (No. 11774423,11822412), the Fundamental Research Funds for the Central Universities, and the Research Funds of Renmin University of China (RUC) (18XNLG14, 19XNLG17). Work at Boston College was supported by the U.S. Department of Energy, Basic Energy Sciences Grant No. DE-FG02-99ER45747. F.C.C. and H.L. acknowledge support from the National Center for Theoretical Sciences and the Ministry of Science and Technology of Taiwan under Grants No. MOST-107-2628-M-110-001-MY3 and MOST109-2112-M-001-014-MY3. F.C.C. is also grateful to the National Center for HighPerformance Computing for computer time and facilities. B.M.A. and H.O.M.S. acknowledge support from the Independent Research Fund Denmark grant number DFF 802100047B.

\section{Author contributions}

J-X.Y., N.S., Y.J., and S.S.Z. conducted the scanning tunneling microscopy experiments in consultation with M.Z.H.; H.Z., S.J., Q.W., and H.Lei synthesized and characterized the magnetic and transport properties of samples; G.M., H.O.M.S., G.C., B.M.A., Z-Q.H., F-C.C., H.Lin, and Z.W. carried out the theoretical analysis in consultation with J-X.Y. and M.Z.H.; Z.C., Z.G., Y.L., M.L., I.B., X.Y., Q.Z., and T.A.C. contributed to the calibration of the measurement; J-X.Y., N.S., Y.J., and M.Z.H performed the data analysis and figure development and wrote the paper with contributions from all authors; M.Z.H. supervised the project. All authors discussed the results, interpretation, and conclusion.

\section{Competing interests}

The authors declare no competing interests.

\section{Additional information}

Supplementary information is available for this paper at https://doi.org/10.1038/s41467020-18111-6.

Correspondence and requests for materials should be addressed to J.-X.Y. or M.Z.H.

Peer review information Nature Communications thanks the anonymous reviewer(s) for their contribution to the peer review of this work.

Reprints and permission information is available at http://www.nature.com/reprints

Publisher's note Springer Nature remains neutral with regard to jurisdictional claims in published maps and institutional affiliations.

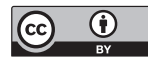

Open Access This article is licensed under a Creative Commons Attribution 4.0 International License, which permits use, sharing, adaptation, distribution and reproduction in any medium or format, as long as you give appropriate credit to the original author(s) and the source, provide a link to the Creative Commons license, and indicate if changes were made. The images or other third party material in this article are included in the article's Creative Commons license, unless indicated otherwise in a credit line to the material. If material is not included in the article's Creative Commons license and your intended use is not permitted by statutory regulation or exceeds the permitted use, you will need to obtain permission directly from the copyright holder. To view a copy of this license, visit http://creativecommons.org/ licenses/by/4.0/.

(C) The Author(s) 2020 